\title{
Research Paper: Normalization of Pervasive Developmental Disorder Screening Test
}

\author{
Mansoureh Karimzadeh ${ }^{1}$, ${ }^{*}$ Ali Reza Baneshi², Mahnaz Dehghan Tezerjani ${ }^{3}$, Zeinab Tayyebi Sough ${ }^{4}$
}

1. Department of Pre-school, University of Social Welfare and Rehabilitation Sciences, Tehran, Iran.

2. Department of Assessment and Measurement, Faculty of Psychology \& Education, Allameh Tabataba'i University, Tehran, Iran.

3. Department of Educational Psychology, Faculty of Psychology and Education, Kharazmi University, Tehran, Iran.

4. Department of Psychology and Teaching Exceptional Children, Faculty of Psychology \& Education, Allameh Tabataba'i University, Tehran, Iran.

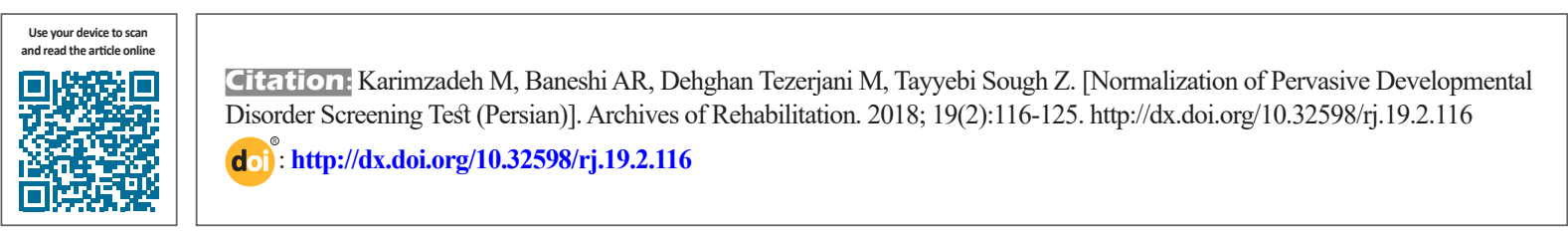

Received: 09 Dec 2017 Accepted: 25 Mar 2018

Keywords:

Standardization,

Screening, Neu-

rodevelopmental disorders, Autism spectrum disorders

\section{ABSTRACT}

Objective According to the Diagnostic and Statistical Manual of Psychiatric Disorders-Fifth Edition, Autism Spectrum Disorders (ASDs) are neurodevelopmental disorders associated with persistent defects in social communication and interactions, patterns, interests, behaviors, and repetitive and limited activities. In other words, ASDs are a lifelong condition characterized by widespread problems in social interaction and communication, stereotypical behaviors and limited interests. The early diagnosis of ASD can lead to early intervention and improvement of developmental outcomes. Parental screening tools are the ideal tools for the timely detection of ASD because they provide diagnostic information from the person familiar with the child and are easy to perform and score. This study aimed to standardize the Pervasive Developmental Disorder Screening Test (PDDST), which screens these disorders at an early age (12 to 48 months). This test consists of 36 questions that are answered as "Yes-No."

Materials \& Methods The population of this descriptive study included all children aged below 8 years with neurodevelopmental disorders and normal children of the same age group in Tehran. In relation to autistic children, the criteria for diagnosis were the persistent impairment of social communication and social interactions in different situations, and patterns of behavior and limited repetitive activities as assessed by a specialist and a psychiatrist. In relation to normal children, the inclusion criteria were the lack of these problems. Thus, a total of 252 children, i.e., 205 children with neurodevelopmental disorders (autism 135 and intellectual disability 70) and 47 normal children (to determine discriminant validity), were included as the sample for this study. Among them, 97 were girls and 157 were boys. The parents of the participants responded to screening tests within 10 minutes at the psychiatric clinics and hospitals in Tehran. The test validity through content by experts and the differential method with variance analysis technique, which shows the significance of the difference between the three groups of participants in the score obtained from the test, and the validity was calculated through the coefficient of internal consistency of Kuder-Richardson 20. Two criteria of validity (sensitivity and specificity) were used to determine the cutting point. Data analysis was performed using the SPSS V. 21 software.

Results The content validity of the test was confirmed according to the experts' judgments. The results of discriminant validity revealed that there is a significant difference between groups $(P=0.001)$. It was also found that the means of normal, intellectual disability, and autistic groups are, respectively, from the lowest to the highest. The reliability of the test was 0.73 . The sensitivity of the child's screening for ASD is indicative of the accuracy of the screening of a child without ASD. The results of this study showed that the PDDST-II screening test for both sensitivity and specificity indices were in a favorable situation and could correctly identify children with autism and children without autistic dysfunction. The two sensitivity and specificity indicators in the score of 5 reached their highest, so the cut-off point for the test was scored at 5 and more.

Conclusion The results of this study showed that the psychometric properties of the PDDST-II screening test are suitable for discriminating between children with ASD and those with other developmental disorders such as mental retardation and normal children. This test also had a good reliability coefficient and is suitable for diagnosing children with ASD.

\section{* Corresponding Author:}

Ali Reza Baneshi, PhD Student

Address: Department of Assessment and Measurement, Faculty of Psychology \& Education, Allameh Tabataba'i University, Tehran, Iran.

Tel: +98 (936) 1349276

E-Mail: alibaneshi4@ut.ac.ir 


\title{
هنجاريابى آزمون غربالكَرى اختلالات طيف اوتيسم
}

\author{
منصوره كريم زاده'ه، "على رضا بانشى'، مهناز دهقان طزرجانى"، زينب طيبى سوق" \\ ا- كروه بيش دبستانى، دانشكاه علوم بهزيستى و توانبخشى، تهران، ايران.

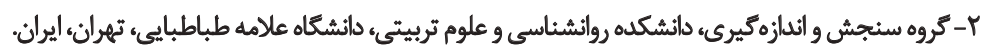

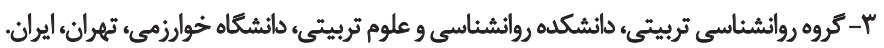

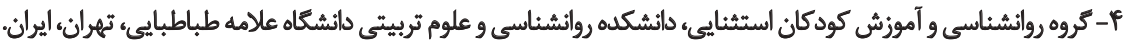

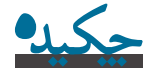

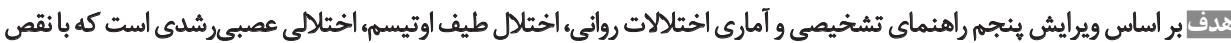

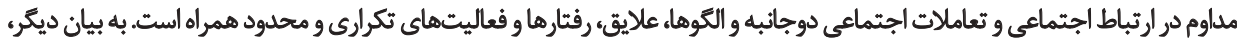

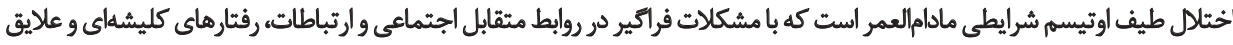

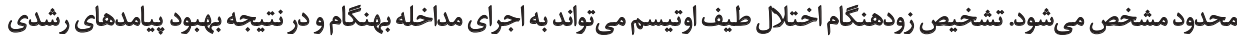

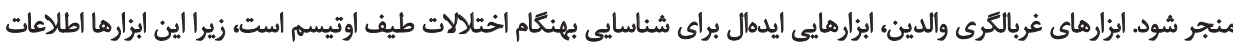

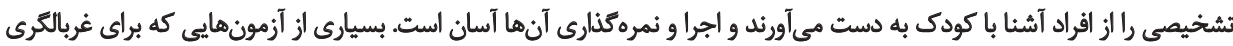

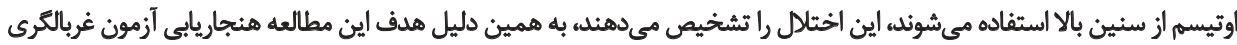

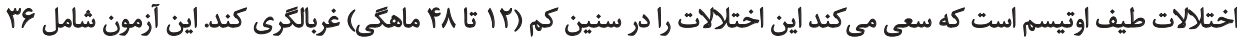

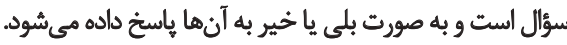

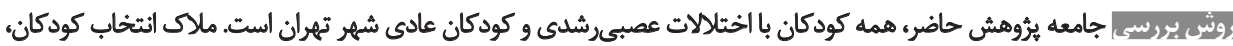

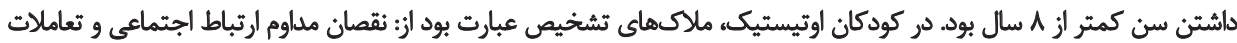

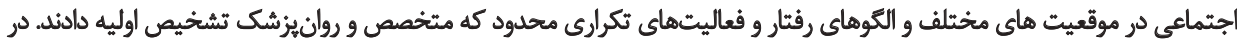

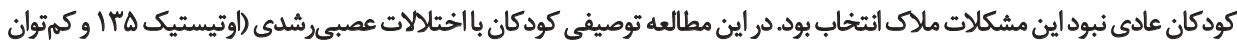

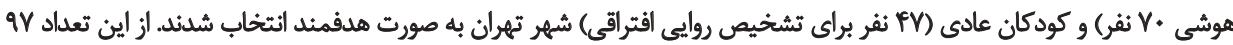

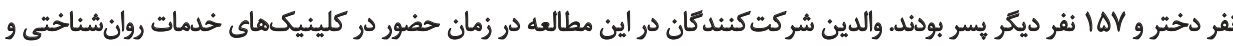

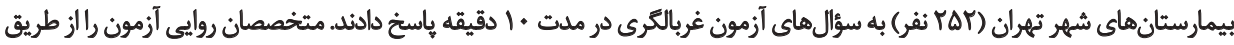

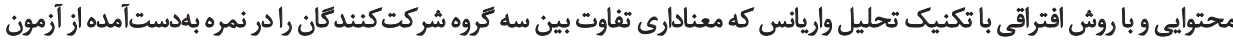

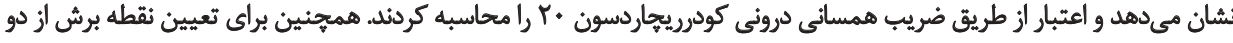

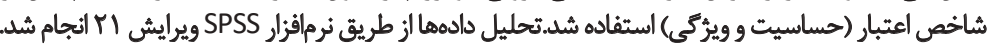

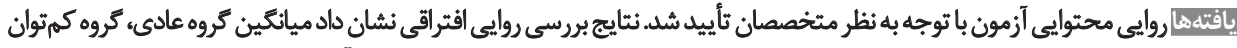

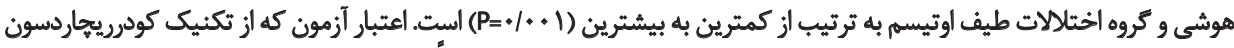

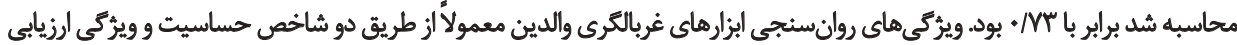

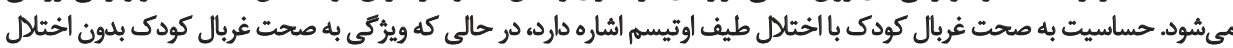

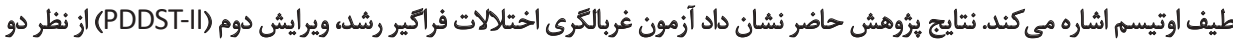

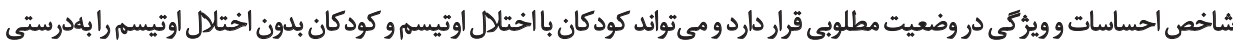

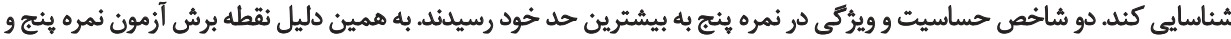

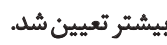

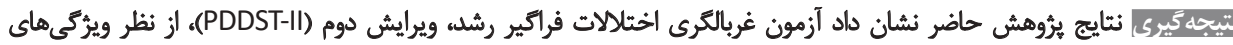

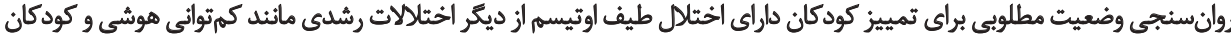

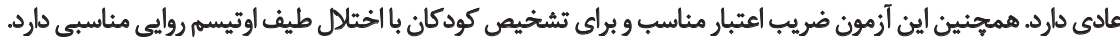

تاريخ دريافت: 14 آذر و

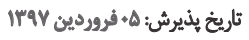

كليدواثرها:

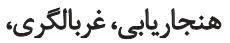

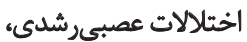
اختلالات طيف اوتيسمر 


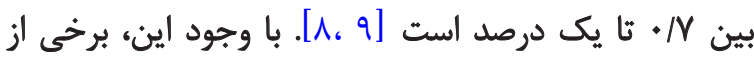

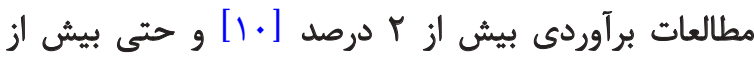

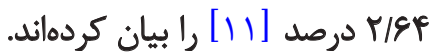

اختلالات طيف اوتيسم در حال حاضر بر اساس ويرايش بنجمي

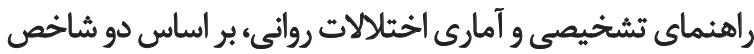

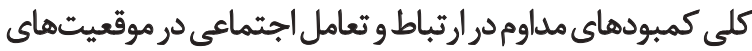

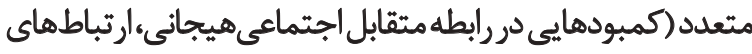

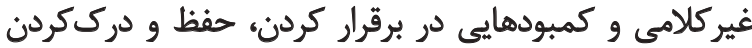

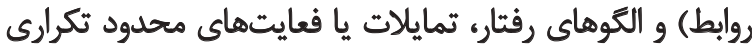

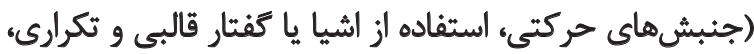

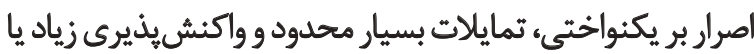

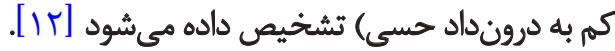
تشخيص اختلال طيف اوتيسم، فرايند بيجيدهائ است كه

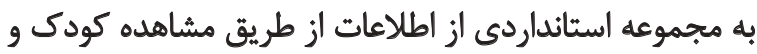

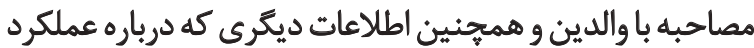

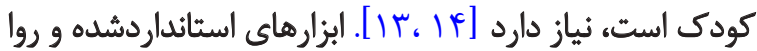

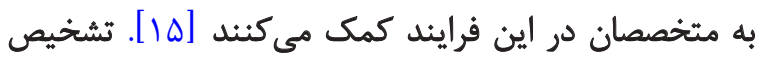

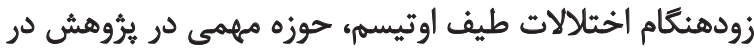

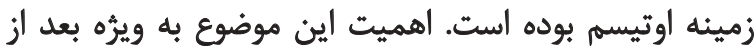

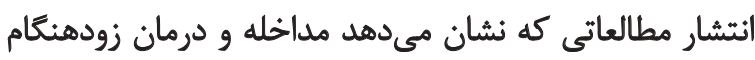

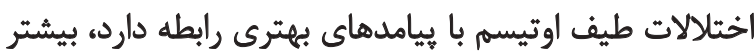

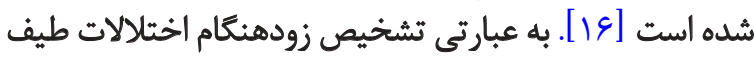

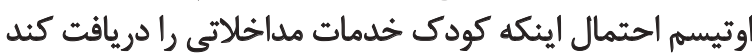

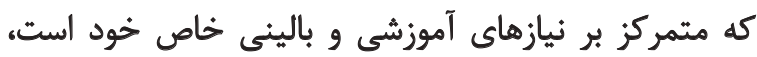

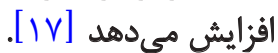

با وجود اينكه اختلالات طيف اوتيسم مىتواند قبل از ازئ

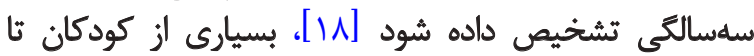

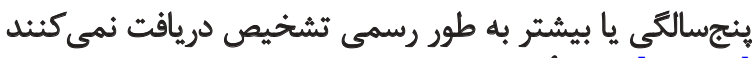

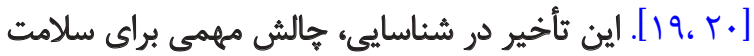

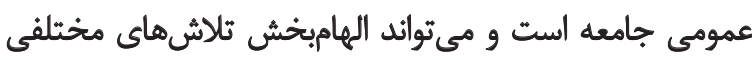

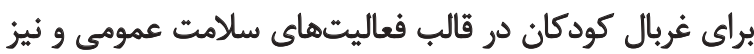

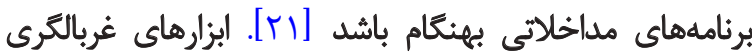

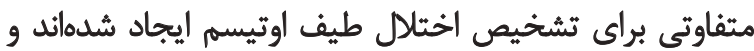

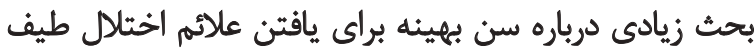

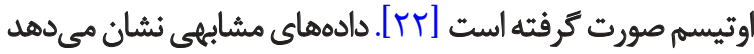

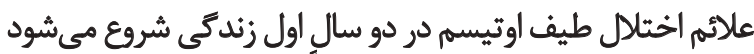

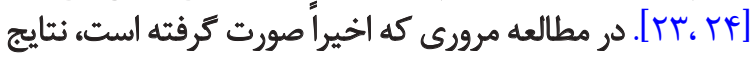

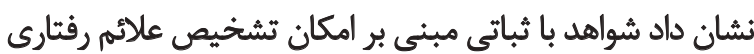

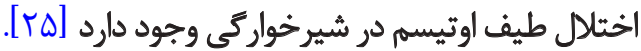
بنابراين، در صورت وجود ابزار غربالكرى اختالل طيف اوتيسم

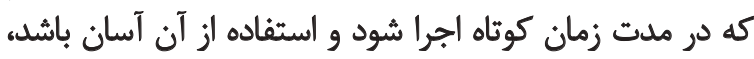

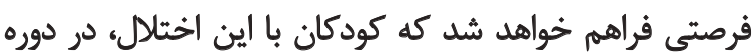

otad

بر اساس ويرايش ئجمم راهنماي تشخيصى و آمارى اختلالات

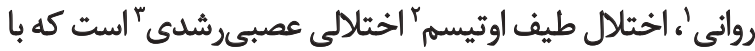

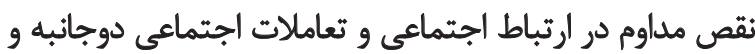

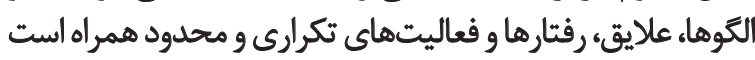

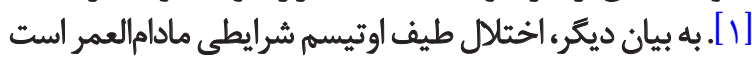

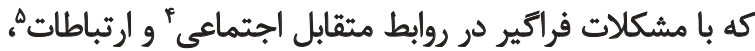

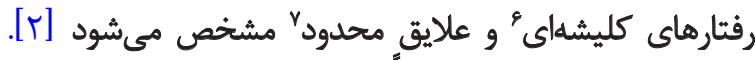

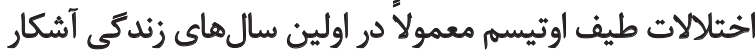

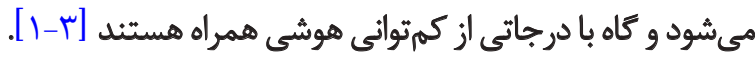

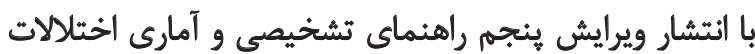

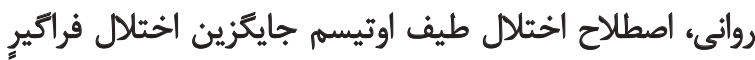

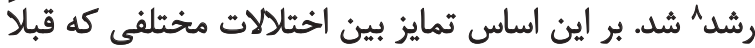

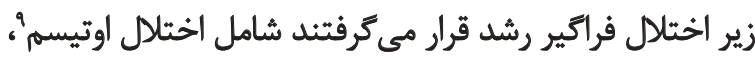

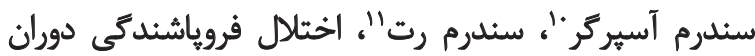

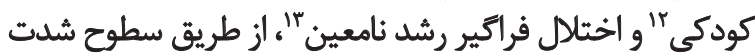

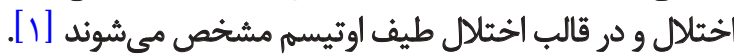

در ساليان اخير شيوع اختلالات طيف اوتيسم (اختلالات

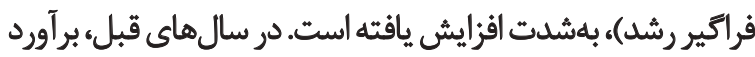

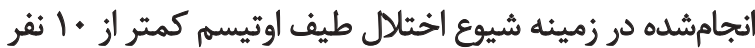

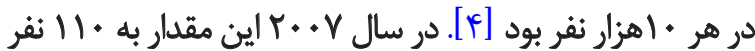

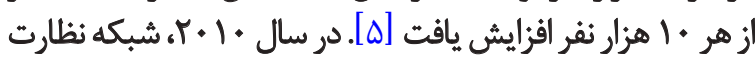

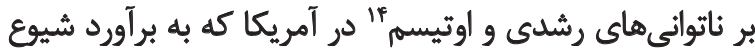

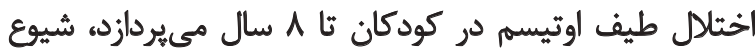

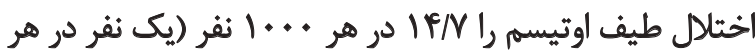

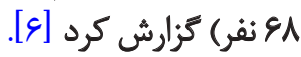

در مطالعهاى كه براى بررسى شيوع اختلالات فراكير رشد در

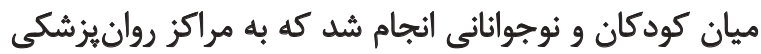

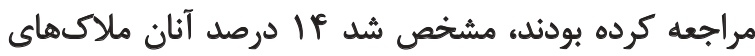

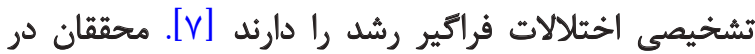

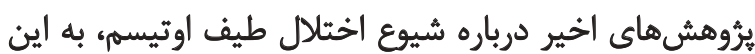

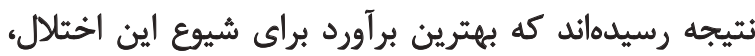

1. Diagnostic and Statistical Manual for mental disorder-fifth edition (DSM-5)

2. Autism Spectrum Disorder (ASD)

3. Neurodevelopmental disorders

4. Social reciprocity

5. Communication

6. Stereotyped behaviors

7. Restricted interests

8. Pervasive developmental disorders

9. Autism

10. Asperger syndrome

11. Rett Syndrome

12. Childhood disintegrative disorder

13. Pervasive developmental disorder not otherwise specified (PDD-NOS)

14. Autism and Developmental Disabilities Monitoring (ADDM) 


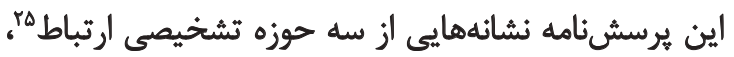

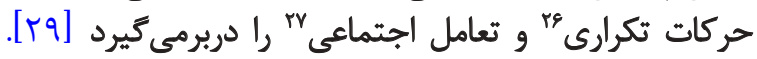

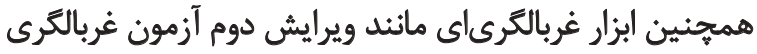

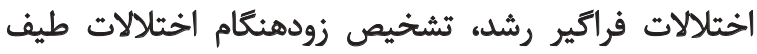

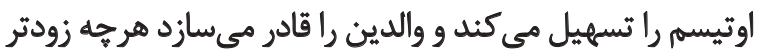

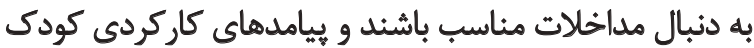

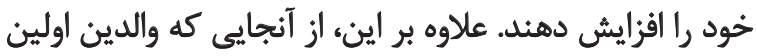

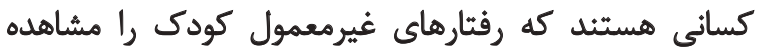

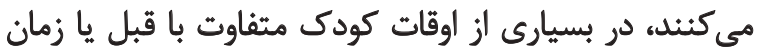

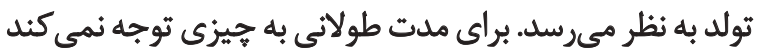

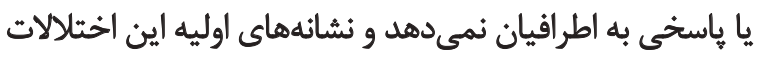

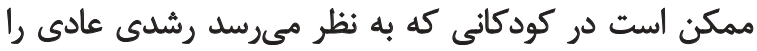

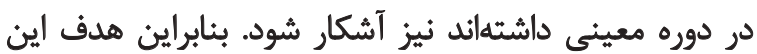

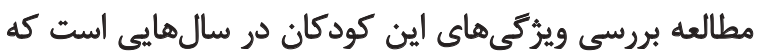

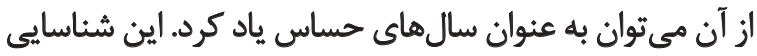

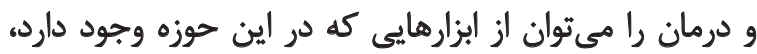

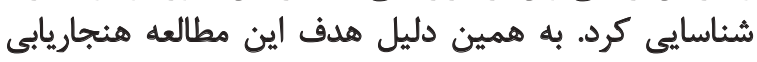

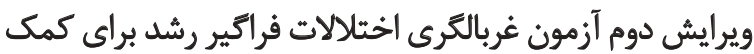
به تشخيص زودهنكام و درمان كودكان است.

\section{ووش بوريى}

روش اين مطالعه توصيفى است. جامعه آمارى اين مطالعه

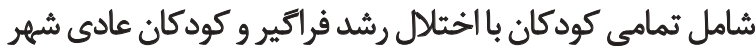

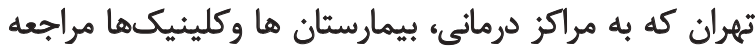

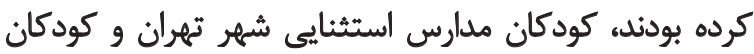

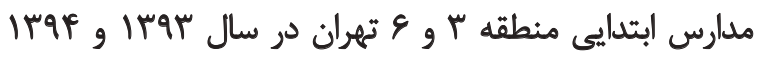

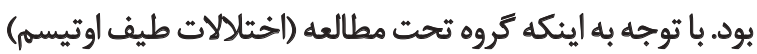

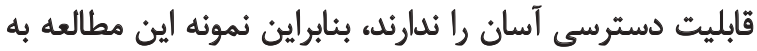

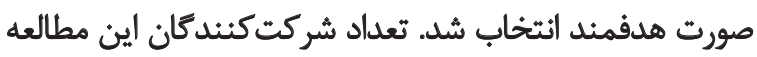

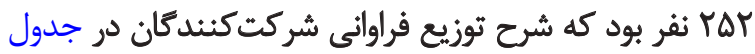

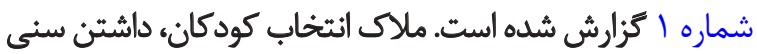

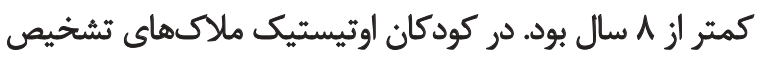

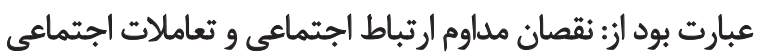

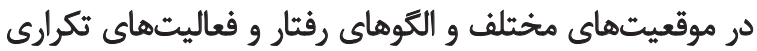

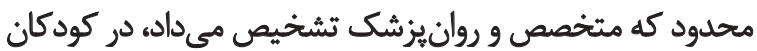
عادى نبود اين مشكلات ملاك انتخاب بود.

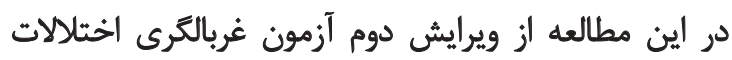

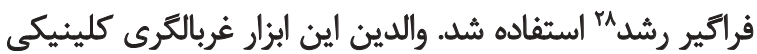

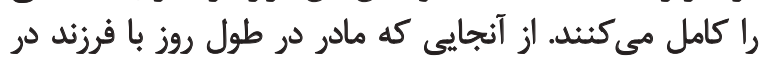

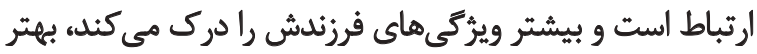

28. Pervasive Developmental Disorders Screening Test (PDDST-II)

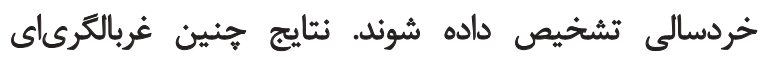

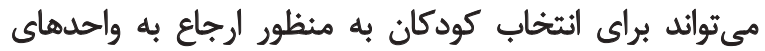

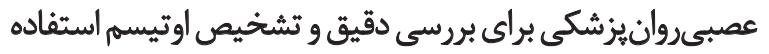

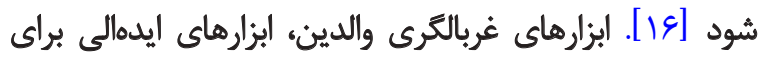

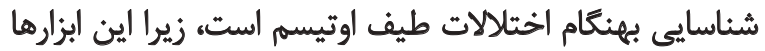

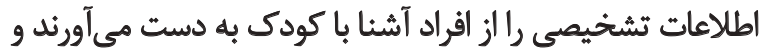

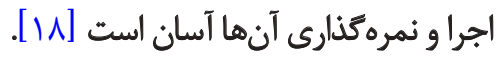
جنانجه اختلالات فراكير رشد بهموقع تشخيص داده شوند،

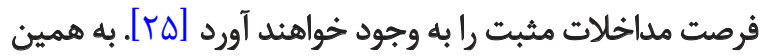

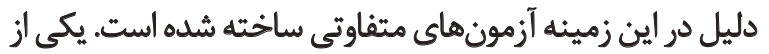

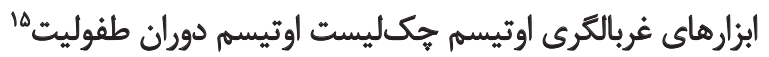

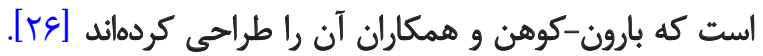

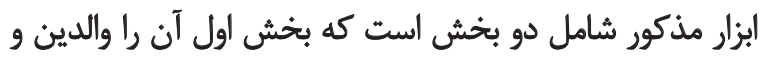

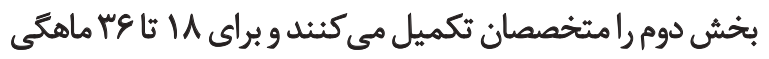

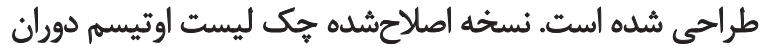

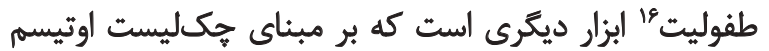

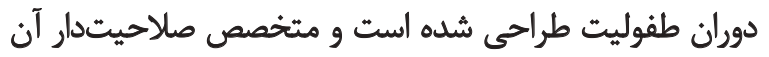

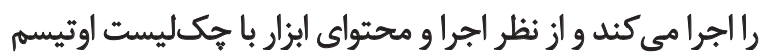

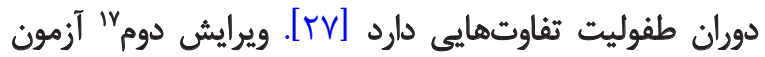

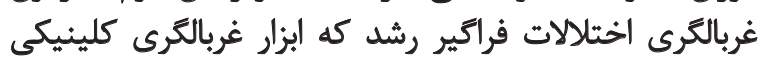

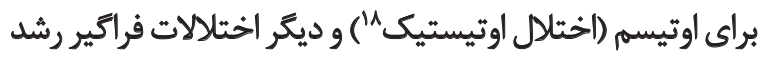

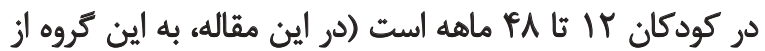

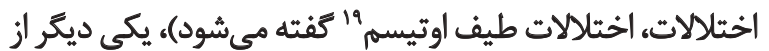

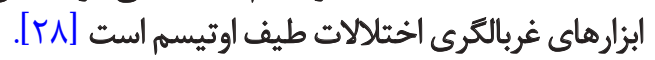

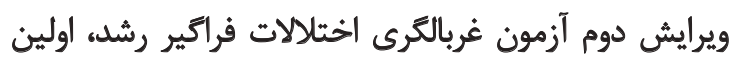

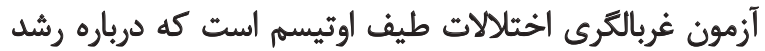

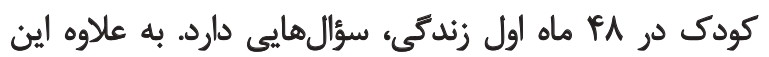

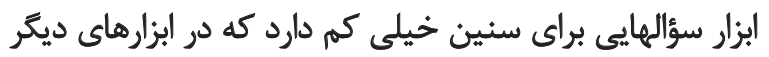

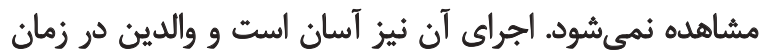

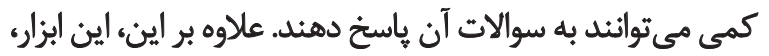

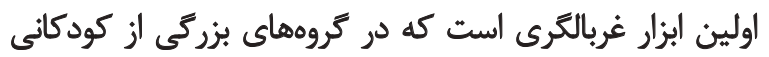

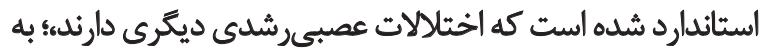

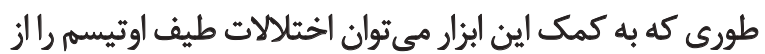

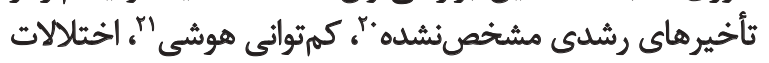

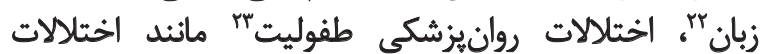

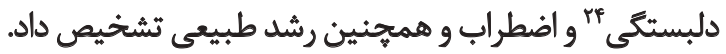

\section{Checklist for Autism in Toddlers (CHAT)}

16. Modified Checklist for Autism in Toddlers (M-CHAT)

17. Pervasive Developmental Disorders Screening Test-II (PDDST-II)

18. Autistic Disorder (AD)

19. Autistic Spectrum Disorders (ASDs)

20. Non-specific developmental delays

21. Intellectual disability

22. language disorders

23. Infant psychiatric disorders

24. Attachment disorders 


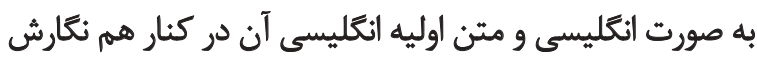

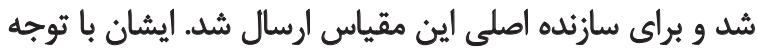

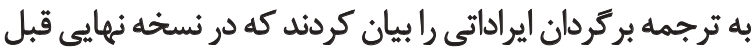

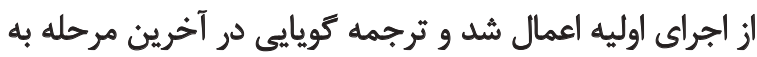
دست آمد.

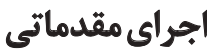

در مرحله اوليه قبل از اجرا از كارشناسان ارشد كودكان

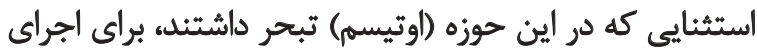

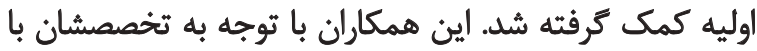

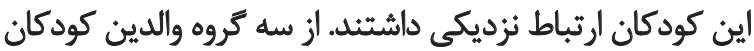

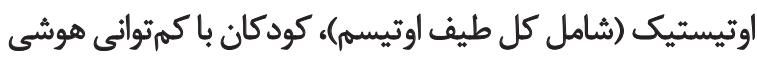

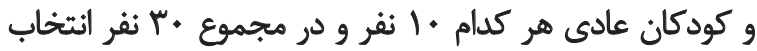

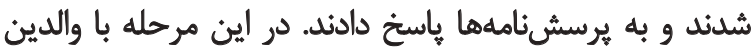

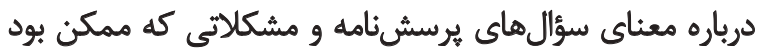

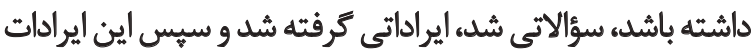

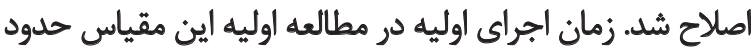

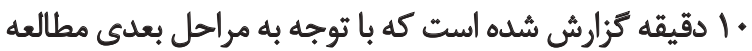

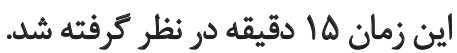

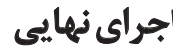

در اجراى نهايى به دو گونه عمل شد. براى جمع آورى اطلاعات

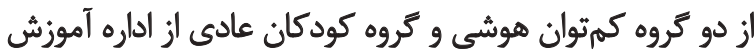

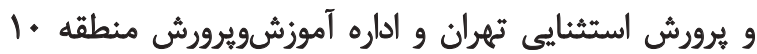

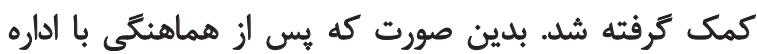

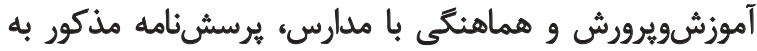

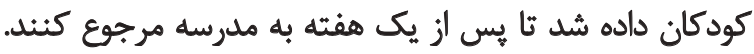

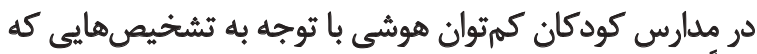

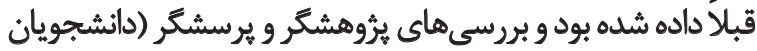

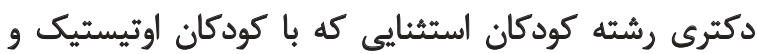

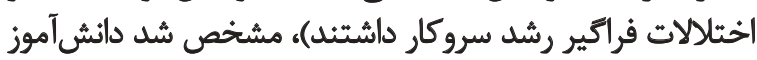

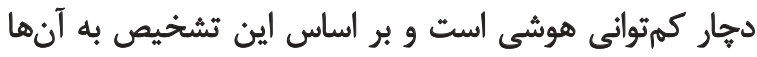
يرسشنامه داده شد.

از آنجايي كه كودكان اختيلالات طيف اوتيسم اهميت خاصى

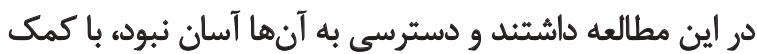

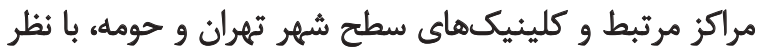

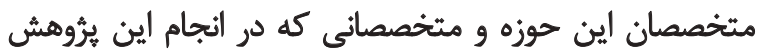

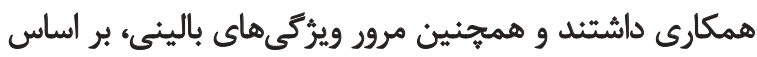

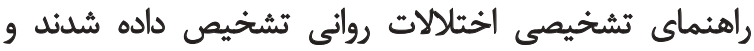

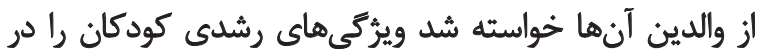

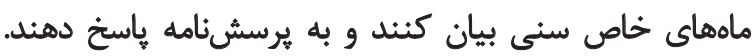
جمعآورى اطلاعات از اين كروهها هفتماه به بون بول انجاميد. والد يا فردى كه با رفتار كودى آشناست، با علامتزدن يانزدن
است او به سؤالات پاسخ دهد. اوتيسم (اختلال اوتيسمى) و ديكر

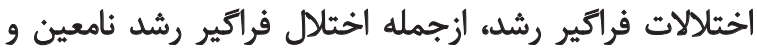

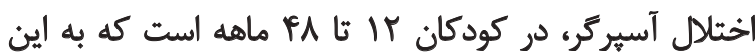

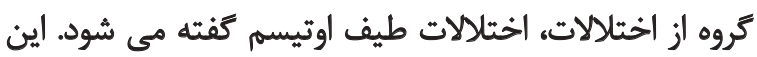

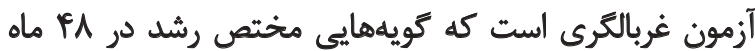

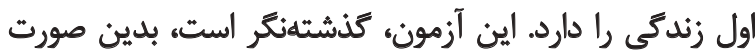

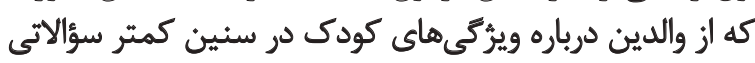

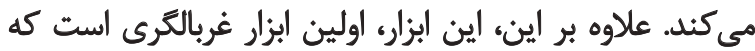

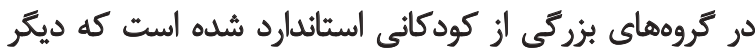

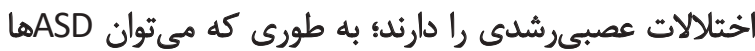

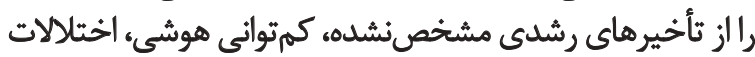

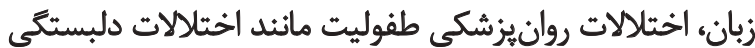

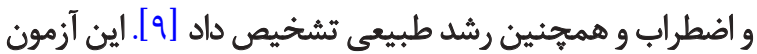

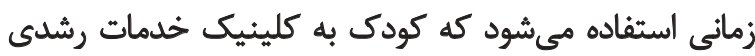

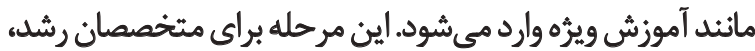

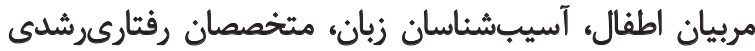

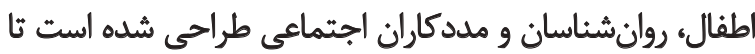

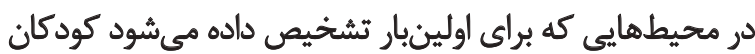
به خدمات تحولى نياز دارند، از آن استفاده كنيند.

در اين مرحله، هدف، تمايز كودكان با ASD از افرادى است

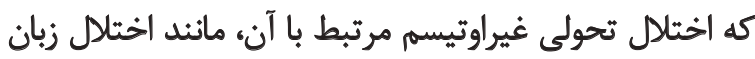

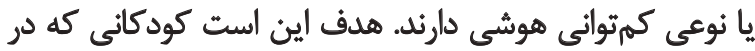

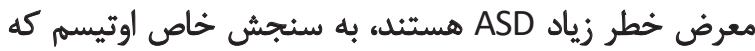

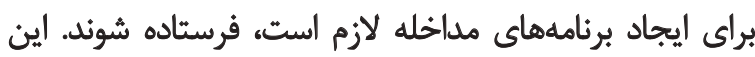

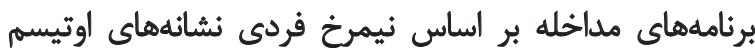

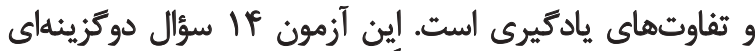

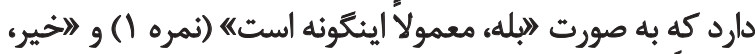

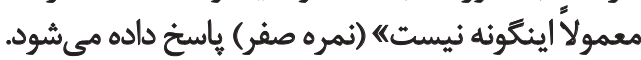

$$
\text { شيوه إمبر }
$$

شيوه اجراى اين يرسشنامه در جند مرحله به صورت زير

$$
\text { صورت يذيرفته است: }
$$

\section{تحليلهاى واضحسازى يرسش نامه}

در بخش اول، يكى از دانشجويان دكترى رشته روانشناسى راني

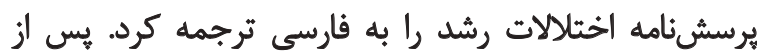

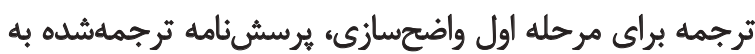

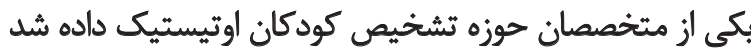

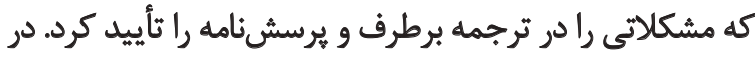

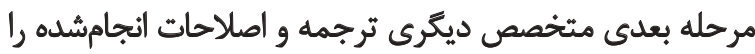

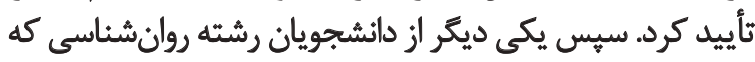

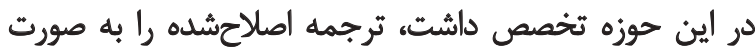
بركردان به انكليسى ترجمه كرد. در مرحله آخر ترجمه انجامشهده آنه 
نتايج بهدستآمده نشان ميدهد ميانكين كروه عادى،

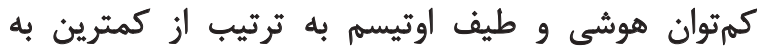

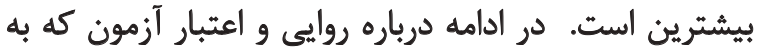

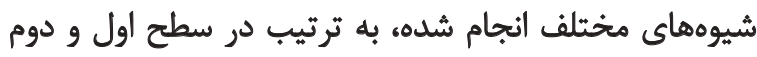

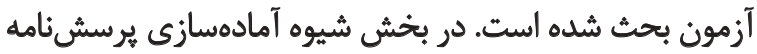

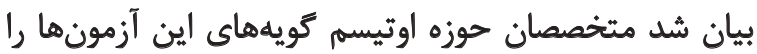

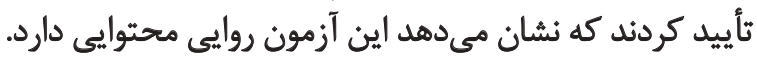

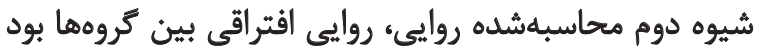

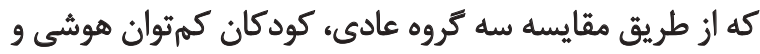

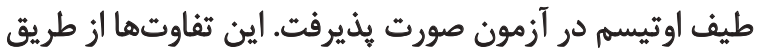

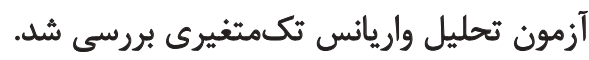

بررسى تفاوت واريانس (آزمون لوين) در بين سه كروه نشان

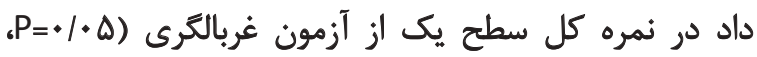

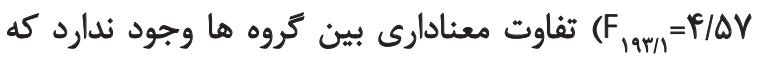

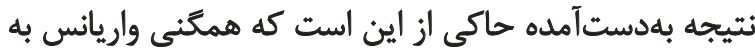

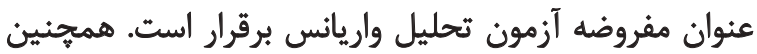

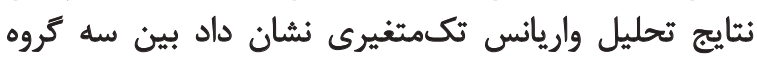

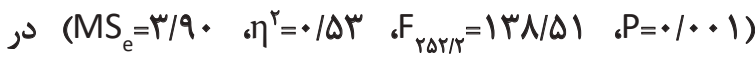

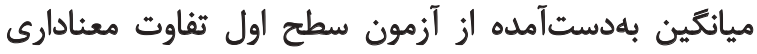

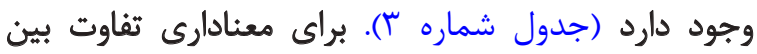

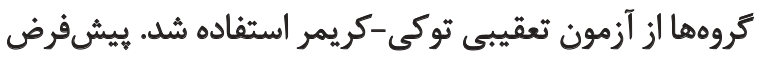

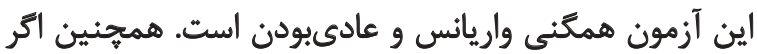

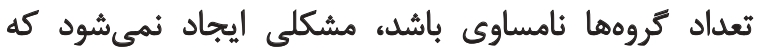

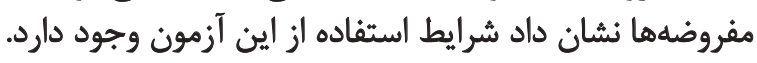
نتايج آزمون تعقيبى در مقابلههاى بين گروهها نشان داد
كويهاي كه در فهرست آمده است و رفتار كودى رادر سن خماصى نشان مى دهد، فرم را تكميل مى كند.

$$
\text { تحليل هاى آمارى }
$$

براي مرحله مقدماتى و تحليل اوليه در اجراي مقدماتى از

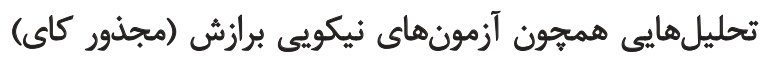

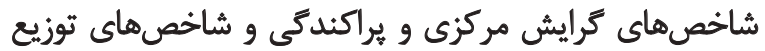

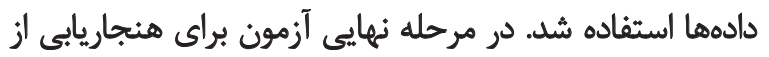

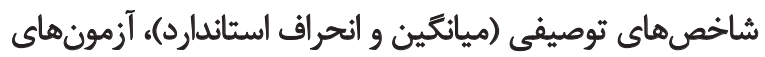

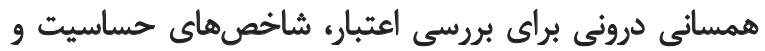
ويثگى و تحليل واريانس استفاده شد. بران

ياقتهنها

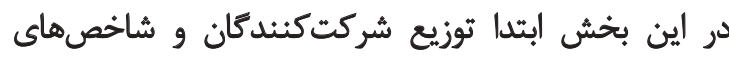

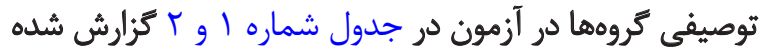

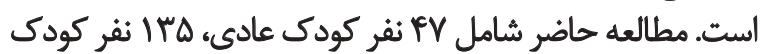

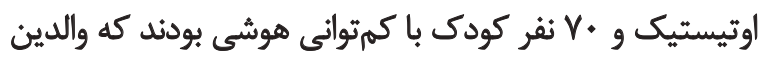

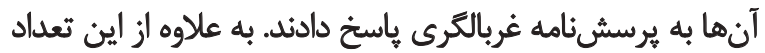

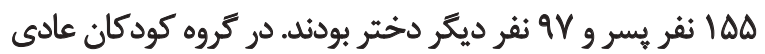

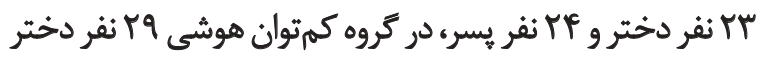

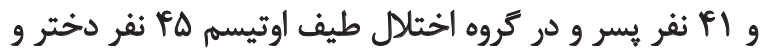

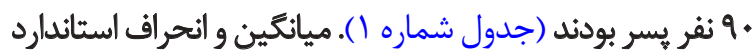

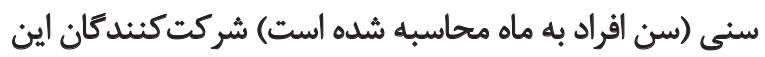

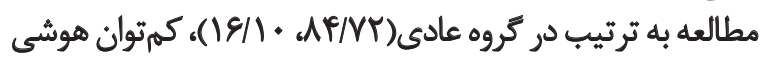
)

جدول ا. توزيع فراوانى شركت كنيدكان

\begin{tabular}{|c|c|c|c|}
\hline كل كل & يسر & دختر & كروه \\
\hline ev & nf & m & كودكان عادى \\
\hline v. & is & rq & كودكان كمثوان هوشى \\
\hline 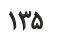 & 9. & Po & الوتيسم \\
\hline TAY & 100 & qv & كل \\
\hline
\end{tabular}

توانبخننى

جدول r. ميانكين و انحراف معيار كروهما

\begin{tabular}{|c|c|c|}
\hline انحراف معيار & مياتكين & كروه \\
\hline $1 / M$ & V/ar & مادى \\
\hline $1 / 9$ & $r / 91$ & كمتوان هوشى \\
\hline$r / \pi$ & $8 / 0$ & طيف اوتيسم \\
\hline
\end{tabular}


جدول ץ. نتايج تحليل واريائس يكراهل بين سه كروه

\begin{tabular}{|c|c|c|c|c|}
\hline حجمه اثثر & $P$ & $\mathbf{F}$ & MS & منيع اثر \\
\hline \multirow[t]{2}{*}{.$/ \Delta r$} & .1 .01 & $|W N D|$ & PTV/aq & بين كروهى \\
\hline & & & $4 / .9$ & درون كروهى \\
\hline
\end{tabular}

$d f i=r d f r=r \Delta r$

جدول F. نتايج آزمون تعقيبى توكى بين كرومها

\begin{tabular}{|c|c|c|c|c|}
\hline \multicolumn{2}{|c|}{ فاصله اطمينان } & اختلاف & كروه & كروه \\
\hline 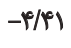 & $-8 / 18$ & $-\Delta / 7 q$ & طيف اوتيسم & عادى \\
\hline$-1 /(\Delta)$ & $-r / \mu$ & $-T / \varphi$ & كمتوان هوشُشى & عادى \\
\hline$r / 9 V$ & $Y /$ & $r / 19$ & كمتوان هوشى & طيف اوتيسم \\
\hline
\end{tabular}

توانبخننى

اختلالات فراكير رشد بود. اين آزمون به دنبال تشخيص كودكان

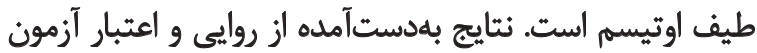

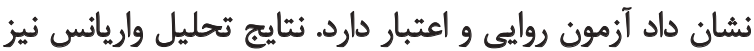

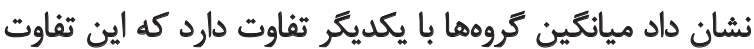

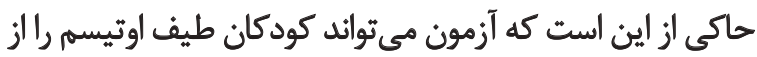

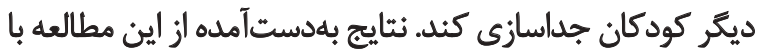

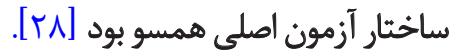

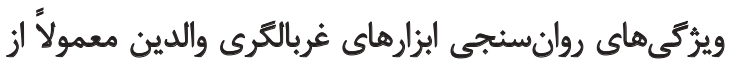

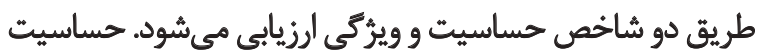

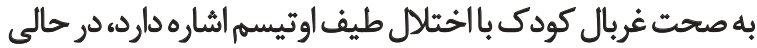

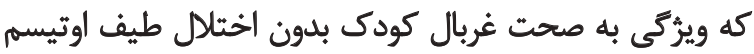

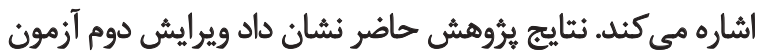

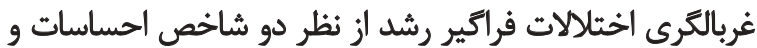

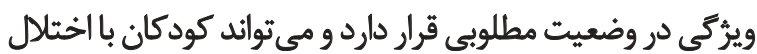

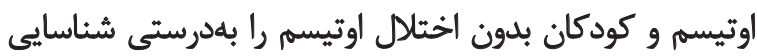

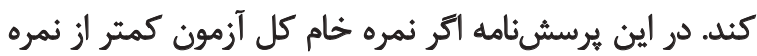

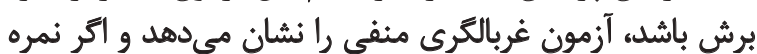

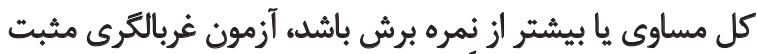

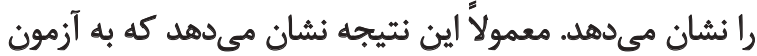

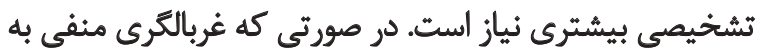

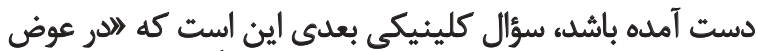

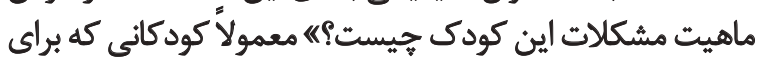

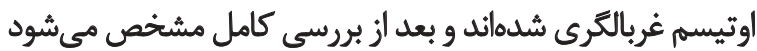

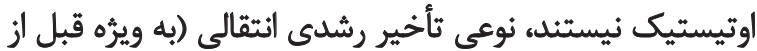

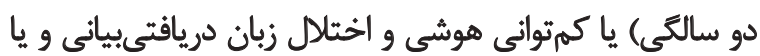

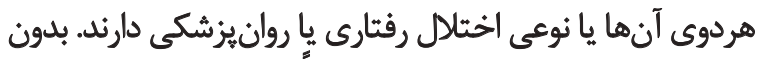

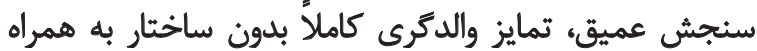

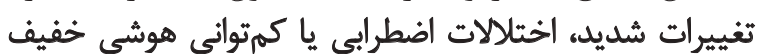

ميانكين كروه عادى از كروه كمتوان هوشى و طيف اوتيسم كمثر،

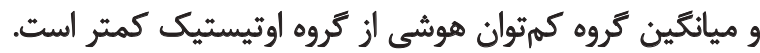

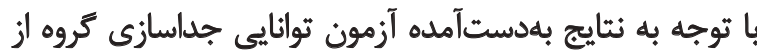

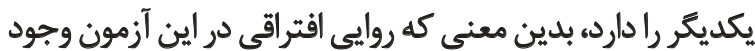

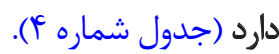

براى بررسى اعتبار آزمون از روش همسانى درونى نارئ

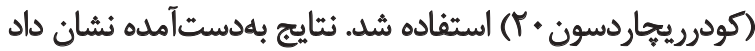

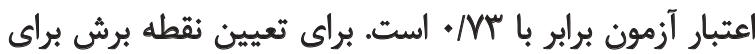

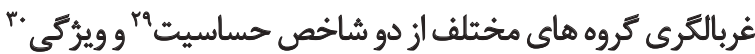

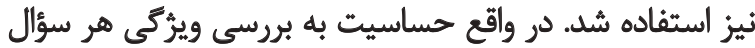

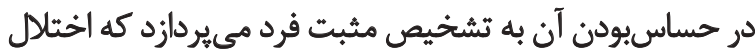
دارد. مقدار اين شاخص بين صفر تا يك است

ويزٔكى عبارت است از قدرت سؤال براى تشخيص صحيح

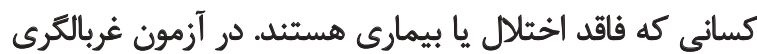

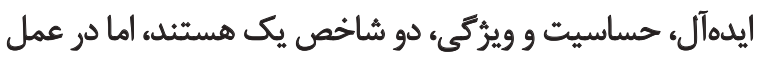

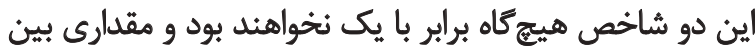

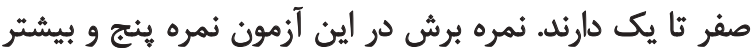

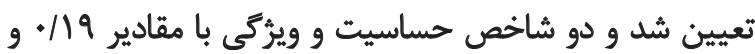

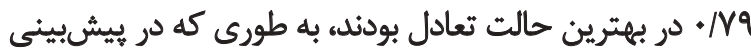

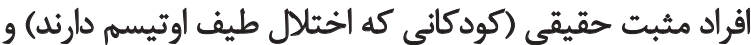

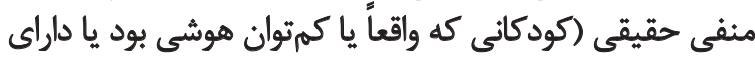
شرايط عادى هستند) بيشترين توانايى را داشت كان. بحث هدف از اين مطالعه هنجاريابى ويرايش دوم آزمون غربالكرى 


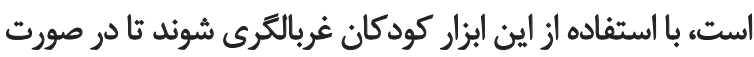

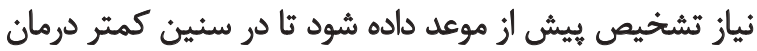

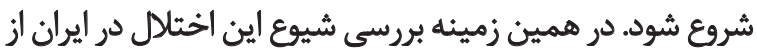

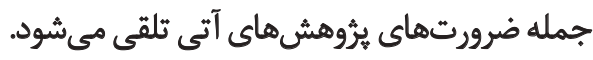

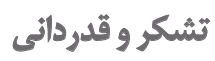

مقاله حاضر بركرفته از طرح برؤوهشى تصويب شده در دانشكاه

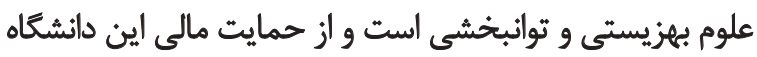

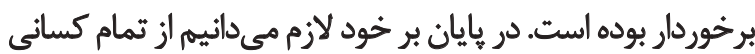

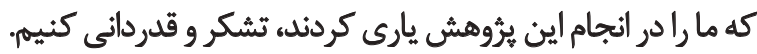

از اختلال طيف اوتيسم، بسيار دشوار است. اين آزمون عنوان

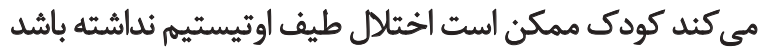

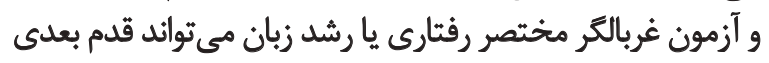

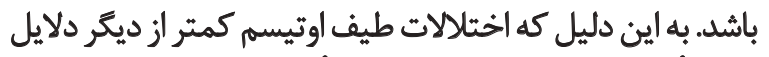

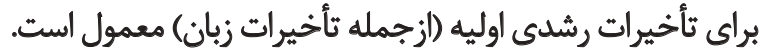

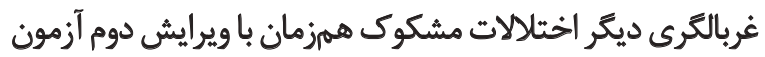

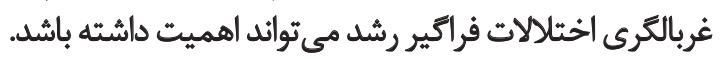

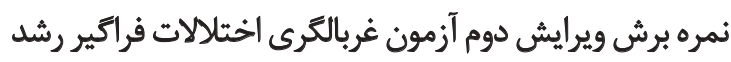

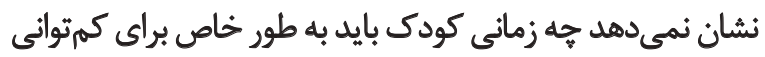

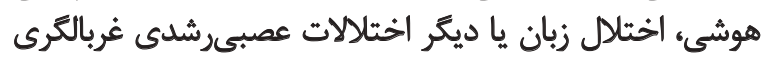

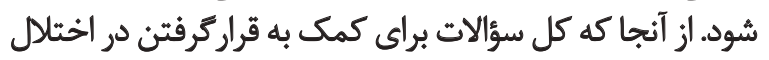

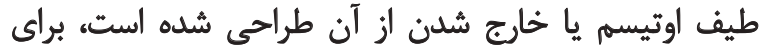

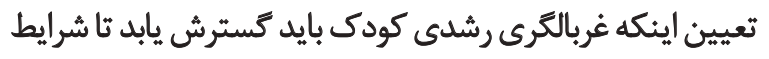

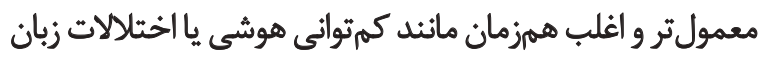

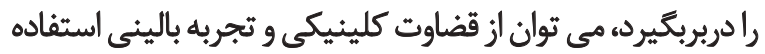

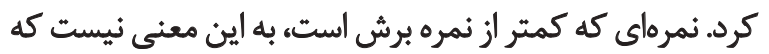

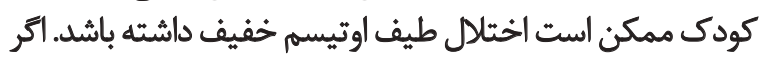

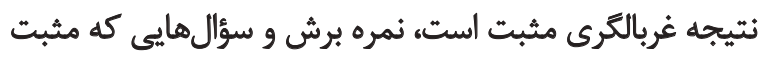

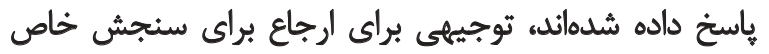
اوتيسم تكميلى هستند.

\section{نتيجه كَيرى}

به طور كلى نتايج يروهش حاضر نشان داد ويرايش دوم آزمون

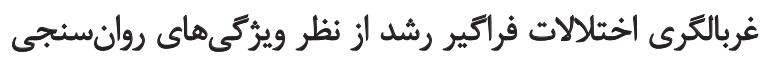

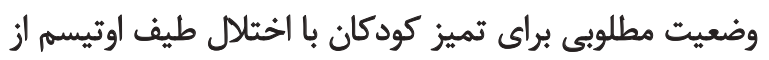

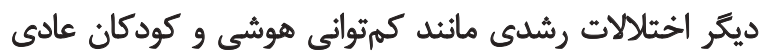

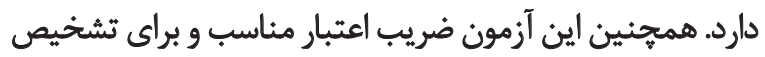

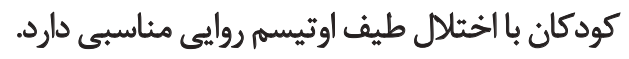

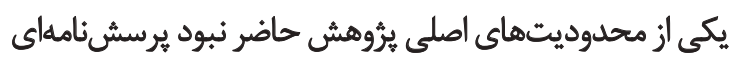

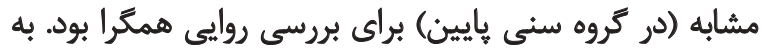

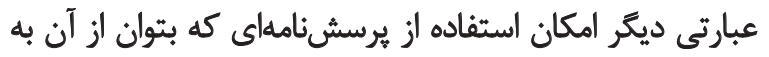

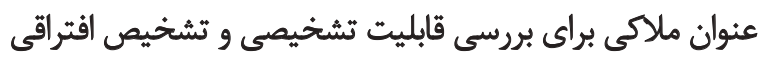

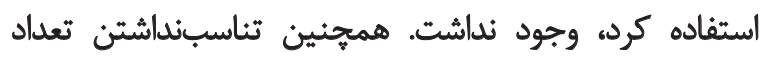

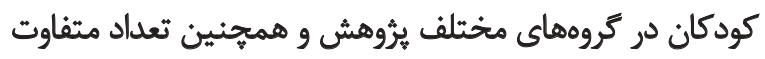

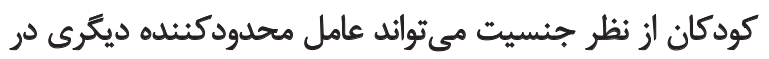

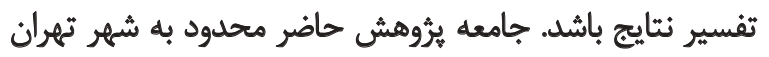

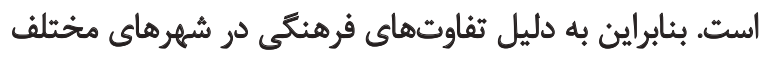
ايران انجام يروهش هاى مشابه در ديكر شهرها مي تواند مفيد دريد باشد.

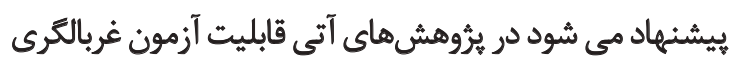

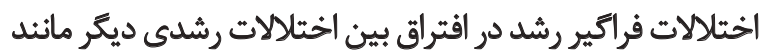

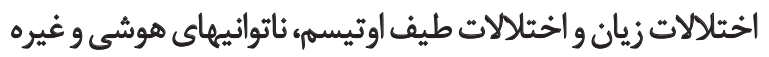

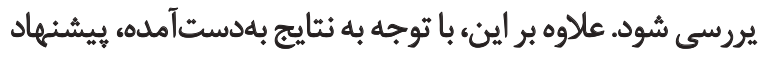

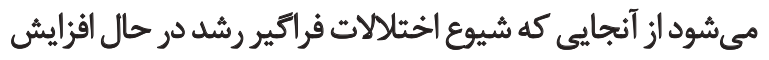




\section{References}

[1] American Psychiatric Association. Diagnostic and statistical manual of mental disorders (DSM-5®). Philadelphia: American Psychiatric Association; 2013.

[2] Shiri V, Hosseini SA, Pishyareh E, Nejati V, Biglarian A. [Study the relationship of executive functions with behavioral symptoms in children with high-functioning autism (Persian)]. Archives of Rehabilitation. 2015; 16(3):208-17.

[3] Bahramkhani M, Darvishi N, Keshavarz Z, Dadkhah A. [The camparison of executive functions in normal and autistic children, considering mathematics and reading abilities (Persian)]. Archives of Rehabilitation. 2013; 13:128-35.

[4] Matson JL, Kozlowski AM. The increasing prevalence of autism spectrum disorders. Research in Autism Spectrum Disorders. 2011; 5(1):418-25. [DOI:10.1016/j.rasd.2010.06.004]

[5] Kogan MD, Blumberg SJ, Schieve LA, Boyle CA, Perrin JM, Ghandour RM, et al. Prevalence of parent-reported diagnosis of autism spectrum disorder among children in the US, 2007. Pediatrics. 2009; 124(5):1395-403. [DOI:10.1542/peds.2009-1522]

[6] Developmental Disabilities Monitoring Network Surveillance Year 2010 Principal Investigators; Centers for Disease Control and Prevention (CDC). Prevalence of autism spectrum disorder among children aged 8 years-Autism and developmental disabilities monitoring network, 11 sites, United States, 2010. MMWR Surveillance Summaries. 2014; 63(2):1-21. [PMCID]

[7] Khushabi K, Pouretemad HR. [Investigating the prevalence of pervasive developmental disorders according to sex in a sample of Iranian children referred to medical-rehabilitation centers and psychiatrics clinics (Persian)]. Avicenna Journal of Clinical Medicine. 2006; 13(1):58-60.

[8] Hill AP, Zuckerman K, Fombonne E. Epidemiology of autism spectrum disorders. In: Robinson-Agramonte M, editor. Translational Approaches to Autism Spectrum Disorder. Berlin: Springer; 2015. [DOI:10.1007/978-3-319-16321-5_2]

[9] Elsabbagh M, Divan G, Koh YJ, Kim YS, Kauchali S, Marcín $\mathrm{C}$, et al. Global prevalence of autism and other pervasive developmental disorders. Autism Research. 2012; 5(3):160-79. [DOI:10.1002/aur.239]

[10] Randall M, Sciberras E, Brignell A, Ihsen E, Efron D, Dissanayake C, et al. Autism spectrum disorder: Presentation and prevalence in a nationally representative Australian sample. Australian \& New Zealand Journal of Psychiatry. 2016; 50(3):243-53. [DOI:10.1177/0004867415595287]

[11] Kim YS, Leventhal BL, Koh Y-J, Fombonne E, Laska E, Lim E-C, et al. Prevalence of autism spectrum disorders in a total population sample. American Journal of Psychiatry. 2011; 168(9):904-12. [DOI:10.1176/appi.ajp.2011.10101532]

[12] Butcher JN, Hooley JM, Mineka SM. Abnormal psychology. New York: Pearson; 2015

[13] Falkmer T, Anderson K, Falkmer M, Horlin C. Diagnostic procedures in autism spectrum disorders: A systematic literature review. European child \& adolescent psychiatry. 2013; 22(6):32940. [DOI:10.1007/s00787-013-0375-0]
[14] Ozonoff S, Goodlin-Jones BL, Solomon M. Evidence-based assessment of autism spectrum disorders in children and adolescents. Journal of Clinical Child and Adolescent Psychology. 2005; 34(3):523-40. [DOI:10.1207/s15374424jccp3403_8]

[15] Slappendel G, Mandy W, van der Ende J, Verhulst FC, van der Sijde A, Duvekot J, et al. Utility of the 3Di short version for the diagnostic assessment of autism spectrum disorder and compatibility with DSM-5. Journal of autism and developmental disorders. 2016; 46(5):1834-46. [DOI:10.1007/s10803-016-2713-9]

[16] Haglund N, Dahlgren S, Källén K, Gustafsson P, Råstam M. The Observation Scale for Autism (OSA): A new screening method to detect autism spectrum disorder before age three years. Journal of Intellectual Disability-Diagnosis and Treatment. 2016; 3(4):230-7. [DOI:10.6000/2292-2598.2015.03.04.9]

[17] Wiggins LD, Bakeman R, Adamson LB, Robins DL. The utility of the Social Communication Questionnaire in screening for autism in children referred for early intervention. Focus on Autism and Other Developmental Disabilities. 2007; 22(1):33-8. [DOI:10 $.1177 / 10883576070220010401]$

[18] Lord C. Follow-up of two-year-olds referred for possible autism. Journal of Child Psychology and Psychiatry. 1995; 36(8):1365-82. [DOI:10.1111/j.1469-7610.1995.tb01669.x]

[19] Sivberg B. Parents' detection of early signs in their children having an autistic spectrum disorder. Journal of Pediatric Nursing. 2003; 18(6):433-9. [DOI:10.1016/S0882-5963(03)00139-8]

[20] Wiggins LD, Baio J, Rice C. Examination of the time between first evaluation and first autism spectrum diagnosis in a population-based sample. Journal of Developmental \& Behavioral Pediatrics. 2006; 27(2):S79-S87. DOI:10.1097/00004703-200604002 00005]

[21] Zwaigenbaum L, Stone W. Early screening for autism spectrum disorders in clinical practice settings. In: Stone W, Charman T, editors. Social \& communication development in autism spectrum disorders: Early identification, diagnosis, \& intervention. New York: Guilford: 2006

[22] Clifford SM, Dissanayake C. The early development of join attention in infants with autistic disorder using home video observations and parental interview. Journal of Autism and Developmental Disorders. 2008; 38(5):791-805. [DOI:10.1007/s10803007-0444-7]

[23] Zwaigenbaum L, Bryson S, Garon N. Early identification of autism spectrum disorders. Behavioural Brain Research. 2013; 251:133-46. [DOI:10.1016/j.bbr.2013.04.004]

[24] Mamaghanieh M, Pour-Etemad HR, Ahmadi F, Khoushabi K. [The effectiveness of picture exchange communication system on behavioral problems of children with Autism (Persian)]. Archives of Rehabilitation. 2011; 12(1):19-26.

[25] Lord C, Corsello C, Grzadzinski R. Diagnostic instruments in autistic spectrum disorders. In: Volkmar FR, Paul R, Rogers SJ, Pelphrey KA, editors. Handbook of Autism and Pervasive Developmental Disorders. Hoboken, New Jersey: John Wiley \& Sons; 2014.

[26] Baron-Cohen S, Wheelwright S, Cox A, Baird G, Charman T, Swettenham J, et al. Early identification of autism by the Checklist for Autism in Toddlers (CHAT). Journal of the Royal Society of 
Medicine. 2000; 93(10):521-5. [DOI:10.1177/014107680009301 007]

[27] Robins DL, Fein D, Barton ML, Green JA. The Modified Checklist for Autism in Toddlers: an initial study investigating the early detection of autism and pervasive developmental disorders. Journal of Autism and Developmental Disorders. 2001; 31(2):131-44. [DOI:10.1023/A:1010738829569]

[28] Siegel B. Pervasive developmental disorders screening TestII(PDDST-II). New York: Pearson; 2004.

[29] Myles BS. Autism spectrum disorders: a handbook for parents and professionals. Westport, Connecticut: Greenwood Publishing Group; 2007. 